\title{
Tool Path Generation Method of Complex Surface 5- axis Machining
}

\author{
Huiying Li and Liangji Chen ${ }^{*}$ \\ School of Mechatronics Engineering, Zhengzhou University of Aeronautics, China \\ ${ }^{*}$ Corresponding author
}

\begin{abstract}
To enhance 5-axis CNC machining precision of complex surfaces with a ball-end cutter, the generation method of cutter contacting (CC) paths is presented in this paper. By means of curvature circle, the feeding length was calculated within allowable error. The step-over in the perpendicular direction of feeding one, was figured out according to the permissible remnant cusps. With the local curvature and the machining error, the step-size was calculated to get the parameter of the next cutter-contacting point. The corresponding spline path of tool center points including knot vector and controlling points was obtained from the cutter contacting points.
\end{abstract}

Keywords-complex surface; tool path; ball-end cutter; 5-axis machining

\section{INTRODUCTION}

When the CAD systems design 3-D model production that has the character of free surface, it usually takes it as a form of Non-Uniform Rational B-Spline(NURBS)[1].When processing this production in the computer numerical control (CNC) machine tool, it must calculate the tool path of free surface which is expressed with NURBS. The current CNC system generally has the function of interpolation of line or arc, so they can only execute the motion instruction of line or arc. In order to process free surface, tool path is generated by some straight segments or arc segments, and then it will generate the corresponding $\mathrm{CNC}$ codes. The way of approximate machining has the following shortcomings: (1) increase approximated segments to improve machining accuracy, and it may lead to large CNC program and heavy system data load; (2) it needs acceleration and deceleration in the interpolation and it will lead to poor consistency of the cutting speed. (3) the way of approximation lost the original NURBS information of surface expression, and it can lead to lower machine accuracy.

5-axis CNC machine tools are widely used in machining dies, molds, turbine blades, and aerial parts. These parts usually have complex geometry and are represented by parametric or sculptured surfaces. The NURBS method[1] is currently used for representing and designing the above surfaces in most commercial computer-aided design and manufacturing (CAD/CAM) systems. If a 5-axis CNC machine tool is applied to machining a part with complex shape, the NURBS surface model of the part is firstly obtained from CAD system and then 5 -axis tool path of cutting tool is generated in CAM system. However, the above tool path generated by CAM system cannot be directly input into 5-axis CNC machine to execute accurate cutting because of local gouging between cutter and workpiece. Tikhon[2] and Cheng[3,4]had presented a surface machining method for ball-end cutting tool, respectively. However, these ball-end cutting methods had disadvantages such as lower manufacturing efficiency and so on. The CAM system, which is of great generality, is not enough flexible to get a gouging-free tool path for a specific part. So, a special 5axis CAM system must be easily obtained. In order to develop a CAM system for special usage, generally, the urgent task is to generating a gouging-free tool path for an arbitrary NURBS surface machining. In 5-axis surface machining, flat-end cutter, whose effective radius is enlarged when it is inclined and tilted, has been mostly used in relative researches [6-9]. In the rest of this paper, representation method of free-form surface using NURBS is introduced and a 5-axis machining method with a flat-end cutter is proposed based on the NURBS surface.

Fortunately, CNC manufacturers have already realized the above-mentioned problems, and provided the direct control function of NURBS track in their high-grade NC systems. These systems can control the tool spline paths [2 4]. However, the generation of NURBS spline path is a difficult problem, its process of internal operation is different from the current generation type of approximate tool path, and it is more difficult to get the more precise NURBS tool path.

It has a detailed discussion about the problem in this paper, and tries to find the method of free surface of NURBS process path.

\section{NURBS EXPRESSION OF COMPLEX SURFACE}

In the Cartesian coordinate system (o-xyz), a free surface can be expressed as the following parameter form

$$
\begin{array}{r}
\boldsymbol{S}(u, v)=[x(u, v), y(u, v), z(u, v)]^{\mathrm{T}}=x(u, v) \boldsymbol{i}+y(u, v) \boldsymbol{j} \\
+z(u, v) \boldsymbol{k} \quad 0 \leq u, v \leq 1
\end{array}
$$

where $\boldsymbol{i}, \boldsymbol{j}, \boldsymbol{k}$ respectively is the unit vector of the direction of $x, y, z ; u$ and $v$ are the parameters. The free curved surface in the (1) can be expressed with NURBS[5]

$$
\frac{\sum_{s=0}^{n} \sum_{t=0}^{m} B_{s, p}(u) B_{t, q}(v) \omega_{s, t} \boldsymbol{T}_{s, t}}{\sum_{s=0}^{n} \sum_{t=0}^{m} B_{s, p}(u) B_{t, q}(v) \omega_{s, t}} \quad 0 \leq u, v_{\leq 1}
$$


where $\boldsymbol{T}_{s, t}$ is control point; $\omega_{i, j}$ is the corresponding weight factor of $\boldsymbol{T}_{s, t} ;(\mathrm{n}+1)$ is the number of control point of $u$ direction and $(\mathrm{m}+1)$ is the number of control point of $v$ direction; $B_{s, p}(u)$ is the B-spline basis function of $u$ direction and $B_{t, q}(v)$ is the B-spline basis function of $v$ direction, $p$ and $q$ are the times.

\section{Generation of Cutter Contacting Point}

The cutter head of ball-end cutter has good adaptability and cutting quality when milling free surface. So it is widely used in the process of $\mathrm{CNC}$ milling. Before getting the $\mathrm{CNC}$ program of ball cutter, it needs to calculate the cutter contacting point of cutter head sphere and free surface, and get the path curve of cutter contacting point.

\section{A. Determine the Track of Original Cutter Contacting Point}

For the complex surface, it can select one of its' border curve $(\boldsymbol{S}(u, 0), \boldsymbol{S}(u, 1)$ and $\boldsymbol{S}(0, v), \boldsymbol{S}(1, v))$ as the path of original cutter contacting point according to specific circumstance, the subsequent calculation is on the basis of the path of cutter contacting point.

It selects $\boldsymbol{S}(u, 0)$ as an example in this paper and chooses $\boldsymbol{S}(0,0)$ as the first cutter contacting point.

\section{B. Calculation of Contiguous Cutter Contacting Point}

On the path curve of cutter contacting point, the distance of contiguous two cutter contacting point is just the feed step length of this cutter contacting point. Therefore, when calculating the next adjacent cutter contacting point with knowing the last cutter contacting point, it should firstly calculate the feed step length. The calculation of feed step length must be based on the allowing manufacturing error.

It is supposed that the current cutter contacting point is the $\boldsymbol{j}$ th cutter contacting point $\boldsymbol{C}_{i, j}$ on the path curve $\boldsymbol{P}_{i}$ of the $i$ th cutter contacting point, and the next adjacent cutter contacting point is $\boldsymbol{C}_{i, j+1}$. As shown in Fig. $1, \boldsymbol{P}_{i}, \boldsymbol{C}_{i, j}$ and $\boldsymbol{C}_{i, j+1}$ are respectively expressed as $\boldsymbol{P}_{i}=\boldsymbol{S}\left(u, v_{i}\right), \quad \boldsymbol{C}_{i, j}=\boldsymbol{S}\left(u_{j}, v_{i}\right)$ and $\boldsymbol{C}_{i, j+1}=\boldsymbol{S}\left(u_{j+1}, v_{i}\right)$ Where $v_{i}$ is constant between 0 and 1 .

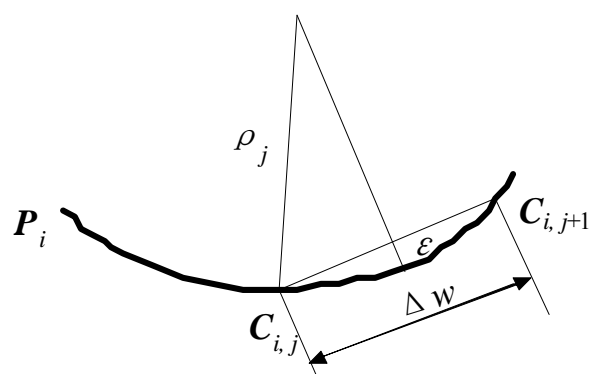

FIGURE I. CALCULATION OF FEED STEP LENGTH

The calculation equation of $\mathrm{C}_{\mathrm{i}, \mathrm{j}}$ curvature which is on the curve $P_{i}$ is

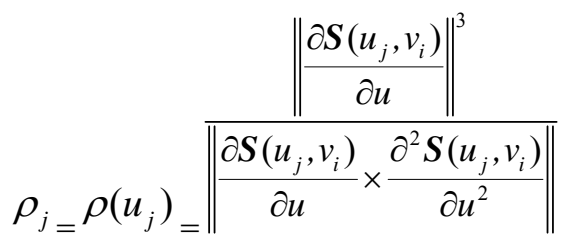

and calculate the feed step length $\Delta w$ according to the following equation

$$
\Delta w=2 \sqrt{\left(\rho_{j}\right)^{2}-\left(\rho_{j}-\varepsilon\right)^{2}}
$$

After calculating $\Delta w$, it can calculate the parameter $u_{j+1}$ of cutter contacting point $C_{i, j+1}$ according to (5)

$$
+\frac{\Delta w}{\left\|\frac{\partial \boldsymbol{S}\left(u_{j}, v_{i}\right)}{\partial u}\right\|}
$$

And then it can calculate the next adjacent cutter contacting point.

\section{Track Calculation of Adjacent Cutter Contacting Point}

Workpiece surface generally is composed of several paths of cutter contacting point in the milling. The distance of adjacent two cutter contacting point path is the process linewidth. For the current cutter contacting point $\boldsymbol{C}_{i, j}$, it is the distance of the cutter contacting point between $\boldsymbol{C}_{i, j}$ and $\boldsymbol{P}_{i+1}$. The path orientation of cutter contacting point is the orientation of parameter $u$ in this paper. So the orientation of process linewidth should be the orientation of parameter $v$.

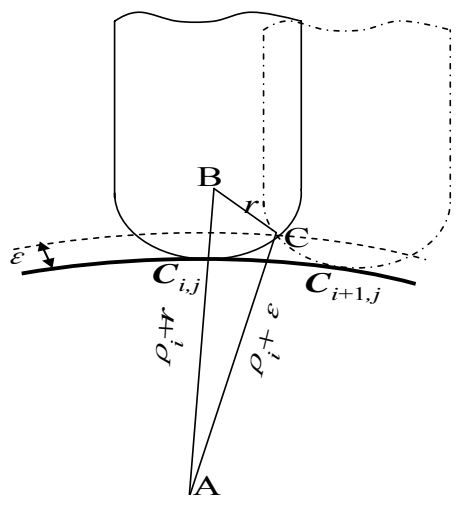

FIGURE II. CALCULATION MATHEMATIC MODEL OF CONVEX SURFACE DISTANCE

As shown in Figure 2, $\rho_{i}$ is the curvature radius that $\boldsymbol{S}(u, v)$ is on the cutter contacting point Ci,j along v direction, $\mathrm{r}$ is the tool bit radius of ball head cutter, $\varepsilon$ is the error that is allowed. Firstly, it can calculate $\rho^{i}$ by 


$$
\rho_{i=} \rho\left(v_{i}\right)_{=} \| \frac{\left\|\frac{\partial \boldsymbol{S}\left(u_{j}, v_{i}\right)}{\partial v}\right\|^{3}}{\left\|\frac{\partial\left(u_{j}, v_{i}\right)}{\partial v} \times \frac{\partial^{2} \boldsymbol{S}\left(u_{j}, v_{i}\right)}{\partial v^{2}}\right\|}
$$

Then the three sides of triangle are all known in Figure 2, and it can conclude the following approximation calculation equation which is about process linewidth according to Helen equation.

$$
\frac{\Delta d_{=}\left\|\boldsymbol{C}_{i, j}-\boldsymbol{C}_{i+1, j}\right\|_{=}}{\frac{2 \sqrt{\left[2 \rho_{i} \pm(2 r+\varepsilon)\right]\left(\rho_{i} \pm \varepsilon / 2\right)\left(\varepsilon r-\varepsilon^{2} / 2\right)}}{\rho_{i} \pm r}}
$$

$$
\Delta d=2 \sqrt{2 \varepsilon r-\varepsilon^{2}}
$$

After calculating $\Delta d$, it can calculate parameter increment in the $\mathrm{v}$ direction by

$$
\Delta v_{j}=\Delta v\left(u_{j}\right)=\frac{\Delta d}{\left\|\frac{\partial \boldsymbol{S}\left(u_{j}, v_{i}\right)}{\partial v}\right\|}
$$

\section{Track Calculation of Adjacent Cutter Contacting Point}

It can conclude generation process of cutter contacting path when machining free curve (as shown in Figure 3):

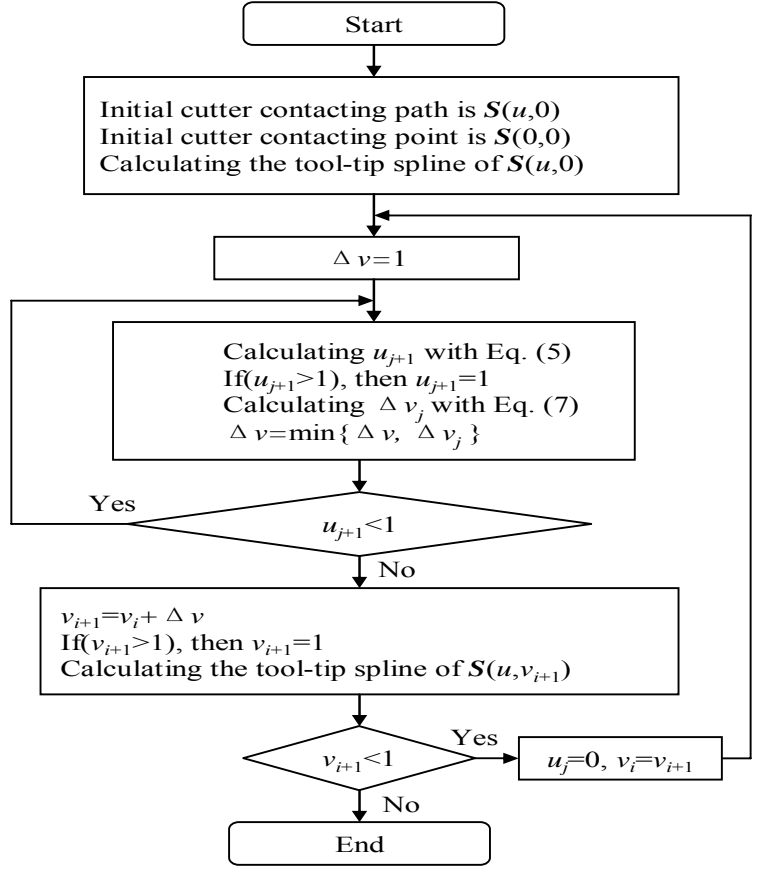

FIGURE III. THE FLOW CHART OF CC PATH GENERATION

\section{GENERATION OF CUTTER CENTER POINT NURBS}

After the generation of all cutter contacting point path of the whole free curved surface, it will generate the $\mathrm{NC}$ process program containing NURBS spline, then calculate cutter center point path that corresponding to every cutter contacting point path $\boldsymbol{S}\left(u, v_{i}\right)$ and signify the curve of cutter contacting point path as NURB spline form.

\section{A. Calculation of Cutter Center Point}

From (2), it can conclude that the control point gridding of $\boldsymbol{S}(u, v)_{\text {is }}\left\{\boldsymbol{T}_{s, t} \mid s=0,1, \cdots, n ; t=0,1, \cdots, m\right\}$. Firstly, for the control point of 0th group and nth group in the u direction, it respectively defines their parameter values as 0 and 1 . So

$$
\hat{u}_{0, t}=0, \quad \hat{u}_{n, t}=1 \quad \mathrm{t}=0,1,2, \cdots, m
$$

Then use the following method to make sure other parameter values of control points

$$
\begin{gathered}
\frac{\left\|\boldsymbol{T}_{l, t}-\boldsymbol{T}_{l-1, t}\right\|}{\hat{u}_{l, t}=\hat{u}_{l-1, t}\left\|\boldsymbol{T}_{k, t}-\boldsymbol{T}_{k-1, t}\right\|} \\
0,1,2, \cdots, m \\
1=1,2, \cdots, n-1 ; \mathrm{t}=
\end{gathered}
$$

Assume that $\left\{\bar{u}_{l} \mid l=0,1, \cdots, n\right\}$ is average parameter values corresponding to $(n+1)$ th control points in the $u$ direction

$$
\bar{u}_{l=} \frac{1}{m+1} \sum_{t=0}^{m} \hat{u}_{l, t} \quad l=0,1, \cdots, n
$$

Input $\bar{u}_{i}$ into (10), and get (n+1) cutter contacting points $\boldsymbol{S}\left(\bar{u}_{l}, v_{i}\right)$ and use the following method to calculate the corresponding cutter center points $\boldsymbol{O}_{l}$

$$
\boldsymbol{O}_{l=} \boldsymbol{S}\left(\bar{u}_{l}, v_{i}\right)_{+\mathrm{r}} \frac{\frac{\partial \boldsymbol{S}}{\partial u} \times \frac{\partial \boldsymbol{S}}{\partial v}}{\left.\left\|\frac{\partial \boldsymbol{S}}{\partial u} \times \frac{\partial \boldsymbol{S}}{\partial v}\right\|\right|_{(u, v)=\left(\bar{u}_{l}, v_{i}\right)}}
$$

\section{B. Vector Calculation of Cutter Center Point}

In the pth order NURB spline, the number of control points is $(n+1)$, and the number of node is $(n+p+2)$. Each node value can be expressed as follows: 


$$
\begin{aligned}
& \tilde{u}_{0}=\tilde{u}_{1}=\cdots=\tilde{u}_{p}=0 \\
& \tilde{u}_{n+1}=\tilde{u}_{n+2}=\cdots=\tilde{u}_{n+p+1}=1 \\
& \tilde{u}_{l+p}=\frac{1}{p} \sum_{i=l}^{l+p-1} \bar{u}_{i} \quad l=1,2, \cdots, n-p
\end{aligned}
$$

\section{Control Point Calculation}

After getting ( $\mathrm{n}+1)$ cutter center points $\boldsymbol{O}_{l}$ from (12), it builds $(n+1) \times(n+1)$ linear equation group about NURBS spline control point Qs.

$$
\boldsymbol{O}_{l=} \sum_{s=0}^{n} N_{s, p}\left(\bar{u}_{l}\right) \boldsymbol{Q}_{s} \quad{ }_{1=} 0,1, \cdots, n
$$

The node vector of primary function $N_{s, p}\left(\bar{u}_{l}\right)$ can be ascertained by (13). There are $(\mathrm{n}+1)$ unknown control points $\boldsymbol{Q}_{\mathrm{s}}$

in (14). The coefficient matrix has the feature of strict opposite angles, and it can get control point $\boldsymbol{Q}_{s}$ by pursued method.

Then it can get NURB spline expression equation of cutter center point after getting control points

$$
\boldsymbol{O}(u)=\sum_{s=0}^{\sum_{s=0}^{n} N_{s, p}(u) \omega_{s} \boldsymbol{Q}_{s}}
$$

\section{CONCLUSION}

It proposes a path generation method of free curved surface expressed by NURBS in this paper. It takes the iso-parametric line on the curved surface as the cutter contacting path between cutter and work-piece. And it takes inside and outside tolerance that is allowed as the reason that calculates feed step length. It also takes request of surface residual high as the calculating reason of line-width. Then it gets the isoparametric line path of the whole curved surface, and finally gets the spline control instruction in the $\mathrm{CNC}$ machining. Comparing with CAM software, the process path which is got by this paper's method has gathered the advantages of isoparametric line and equal residual high. Then it also supplies strong support for NURBS control process function in the CNC system.

\section{ACKNOWLEDGMENT}

This work was financially supported by the National Natural Science Foundation of China(51275485) and Science
\& Technology Innovation Talents in Universities of Henan Province (13HASTIT036).

\section{REFERENCES}

[1] M. Tikhon, T. J. Ko, S. H. Lee and H. S. Kim, "NURBS Interpolator for Constant Material Removal Rate in Open NC Machine Tools," International Journal of Machine Tools \& Manufacture, vol. 44, pp. 237245, 2004

[2] M. Y. Cheng, M. C. Tsai and J. C. Kuo, "Real-time NURBS Command Generators for CNC Servo Controllers,'International Journal of Machine Tools \& Manufacture, vol. 42, pp. 801-813, 2002.

[3] M. C. Tsai, C. W. Cheng and M. Y. Cheng, "A Real-time NURBS Surface Interpolator for Precision Three-axis CNC Machining," International Journal of Machine Tools \& Manufacture, vol. 43, pp. 1217-1227, 2003.

[4] Lin Rongshine, "Real-time Surface Interpolator for 3-D Parametric Surface Machining on 3-axis Machine Tools", International Journal of Machine Tools \& Manufacture, vol. 40, pp. 1513-1526, 2000.

[5] L. Chen, "NURBS Method and Its Application in Location and Federate Controlling System," International Journal of Engineering and Industries, Vol. 2, No. 1, pp. 44-50, 2011.

[6] J. Lai, K. Lin, S. Tseng and W. Ueng, "On the Development of a Parametric Interpolator with Confined Chord Error, Feedrate, Acceleration and Jerk," International Journal Advanced Manufacturing Technology, vol. 37, pp.104-121, 2008.

[7] M. Tsai, H.i Nien, H. Yau, "Development of an Integrated Look-ahead Dynamics-based NURBS Interpolator for High Precision Machinery," Computer Aided Design, vol. 40, pp. 554-566, 2008.

[8] S. Tseng, K. Lin, J. Lai, W. Ueng, "A NURBS Curve Interpolator with Jerk-Limited Trajectory Planning," Journal of the Chinese Institute of Engineers, vol. 32, no. 2, pp. 215-228, 2009.

[9] L. Chen, H. Li and Z. Zhang, "Motion Instruction Generation along Two NURBS Curves in Each Cycle Period," International Journal of Digital Content Technology and its Applications, Vol. 5, No. 7, pp. 350-358, 2011. 